\title{
Dark matter comes in from the cold
}

\author{
The year started with a bang for astronomers and cosmologists gathered at Phoenix, Arizona, last week, with the \\ report of observations indicating that the Universe is made largely of exotic dark material.
}

Humble baryons, the nuclear material from which our Solar System is made, represent a minuscule fraction, about 4 per cent, of the matter in the Universe. Most of the matter is dark, detectable by its gravitational effects alone. Thus runs the recurrent theme of the Big Bang cosmology to which most astrophysicists subscribe. Observational astronomers, however, urge caution, for solid evidence has remained elusive.

But 1993 has begun with release of new observations that promise to resolve some aspects of the controversies still endemic to the Big Bang cosmology. For the first time, diffuse hot intergalactic gas has been detected, by the ROSAT $\mathrm{X}$-ray satellite, in a small group of galaxies (J. S. Mulchaey et al. Astrophys. J. Lett., in the press). As described by Mulchaey in Phoenix last week* ${ }^{*}$, the gas distribution is centred on the group, and the gas temperature is about 10 million degrees kelvin. Knowledge of the gas temperature with the reasonable assumption that the gas is gravitationally confined allows an estimate of the total mass of the group. Baryons, seen as hot gas and in the luminous galaxies, amount to about 4 per cent (and at most 15 per cent) of the total mass. The remainder is dark matter. For the first time, one can conclusively state that diffuse dark matter dominates the gravitational potential of galaxy groups on megaparsec scales. The more dramatic consequence, however, is that we can now aspire to ascertain the nature of the dark matter.

ROSAT's X-ray scan of the NGC2300 group gives not only the gas temperature but also the abundance of heavy clements, formed in stars long after the Big Bang. The spectrum does not have enough resolution to detect individual elements, but taking the element ratios to be solar, Mulchaey and colleagues find the abundance of elements heavier than lithium to be about 6 per cent (and no more than 20 per cent) of their abundance in the Sun. Therefore the gas must be predominantly primordial and predates the galaxies.

Nucleosynthesis in the first few minutes of the Big Bang provides a remarkably concordant explanation of the abundances of the light elements: helium, deuterium and lithium. Theory

*American Astronomical Society, 181st National Meeting. Phoenix, 3-7 January 1993. and observation coincide only if the baryon density in the Universe lies within a narrow range: between 4 and 8 per cent of the dark-matter density in a universe at critical density. New evidence that the Universe is indeed at a critical density, destined to expand forever, but at ever decreasing speed, is presented by K. Kellermann elsewhere in this issue (see the News and Views article by $\mathrm{P}$. Scheuer, overleaf).

Hitherto, astronomers have been able to compare gas and dark matter over large scales only in rich galaxy clusters, where they find diffuse intergalactic gas amounting to at least 10 and even 30 per cent of the dark-matter density in some cases. But that gas is far from pristine, being highly contaminated by ejecta from galaxies - astronomers eagerly await the scheduled launch of the Japanese X-ray satellite ASTRO-D next month to help ascertain the precise location within the clusters of the enriched gas component. Moreover, the complex dynamics of gravitational collapse may operate differentially on gas and dark matter: the X-ray surface brightness and the clumpy distribution of some rich clusters point to this, as does the mapping of dark matter by gravitational lensing. The gas content of rich clusters, even on their outskirts, need not be a good measure of the initial ratio of baryons to dark matter. Only groups of galaxies like NGC2300 can do the trick.

Could the nucleosynthesis prediction be in error? There is of course the cosmological deduction that only a small number (less than four) types of neutrinos are allowed for a unique and simple fit to observations of light-clement abundances. The LEP experiments at CERN, as well as results from the Stanford Linear Collider, converged on precisely three types of neutrinos from studies of neutral Z-boson decays. The prediction also relies on the Hubble constant, measuring the expansion of the Universe, and on the temperature of the cosmic microwave background. Only a Hubble constant of about $50 \mathrm{~km} \mathrm{~s}^{-1}$ $\mathrm{Mpc}^{-1}$ yields an age for the Universe that is consistent with a universe at the critical density and with the ages of the oldest stars. The direct measures of the Hubble constant (involving supernovae, gravitational lensing, microwave background 'absorption' towards galaxy clusters) all favour such a low value, although indirect techniques mostly indicate a somewhat higher value.

The cosmic microwave background is the remaining ingredient. Its temperature has now been measured to be that of a blackbody with deviations no larger than two-hundredths of one per cent near the peak intensity at a wavelength of $1 \mathrm{~mm}$. The temperature is measured to be $2.726 \mathrm{~K}$, with an uncertainty of only $0.01 \mathrm{~K}$, as reported at Phoenix by John Mather, project scientist for the COBE satellite. Such precision in a cosmological measurement is unprecedented. Combination of the microwave background temperature, the Hubble constant and the observations of the light-element abundances leads to an unambiguous prediction of the baryon fraction. Its measurement in diffuse hot gas that is largely uncontaminated by gas stripped from galaxies points towards a Universe at critical density.

The dark matter is the stuff of particle physicist's dreams, some yet-to-bediscovered form of nonbaryonic matter. Examples abound: from axions, weighing in at $10^{-14}$ proton masses to supersymmetric relics such as photinos of 100 proton masses or more. We may have to wait until the next millennium for the Superconducting Super Collider and CERN's Large Hadron Collider to suggest anything more definitive. Or perhaps the particles, if they constitute some large fraction of the dark matter of our own galactic halo, will be detected directly.

Nor need seekers of baryonic dark matter be unhappy: the mass detected in luminous baryonic matter - stars and gas - amounts to at most one per cent of the critical cosmological density. The confirmation of a baryon-to-dark-matter fraction of a few per cent, which must await further ROSAT mapping of other galaxy groups, would practically guarantee that the dark halo of our Galaxy is teeming with compact baryonic fragments, most probably aborted stars, brown dwarfs or stellar remnants (old, cold white dwarfs). We can be certain that dark matter is out there, in amounts on the largest scales that totally dominate the dim glimmer of distant galaxies.

Joseph Silk

Joseph Silk is in the Departments of Astronomy and Physics, University of California, Berkeley, California 94720, USA. 